\title{
Hiệu quả giao tiếp thông tin của doanh nghiệp và hành vi tham gia đồng tạo sinh giá trị của khách hàng: Vai trò của nguồn lực tương tác của khách hàng
}

\section{Communication effectiveness of firm and customer participation behaviors to co-create value: The role of customer operant resources}

\author{
Nguyễn Văn Tuấn ${ }^{1 *}$, Hàng Lê Cẩm Phương ${ }^{1}$, Nguyễn Hồng Đức ${ }^{1}$ \\ ${ }^{1}$ Trường Đại học Bách Khoa - Đại học Quốc gia TP.HCM, Việt Nam \\ "Tác giả liên hệ, Email: nvtuan@hcmut.edu.vn
}

THÔNG TIN

DOI: $10.46223 /$ HCMCOUJS. econ.vi.14.3.474.2019

Ngày nhận: 07/08/2019

Ngày nhận lại: 30/08/2019

Duyệt đăng: 03/09/2019

Tù khóa:

doanh nghiệp, hành vi tham gia đồng tạo sinh giá trị, hiệu quả giao tiếp thông tin, khách hàng, nguồn lực tương tác

Keywords:

communication effectiveness, customer operant resources, customer participation behaviors, customer, firm
TÓM TẮT
Giá trị được đồng tạo sinh và được xác định bởi khách hàng. Nguồn lực tương tác (operant resource) của khách hàng có vai trò quan trọng đối với hoạt động đồng tạo sinh và xác định giá trị dịch vụ. Nguồn lực tương tác của khách hàng được cấu thành bởi 3 dạng là nguồn lực văn hóa, nguồn lực xã hội và nguồn lực vật lý. Kết quả nghiên cứu này chỉ ra rằng trong các tương tác của quá trình cung cấp dịch vụ, doanh nghiệp có cơ hội ảnh hưởng đến nguồn lực văn hóa và nguồn lực vật lý của khách hàng thông qua giao tiếp thông tin hiệu quả. Đây cũng là hai dạng nguồn lực ảnh hưởng tích cực đến hành vi tham gia đồng tạo sinh giá trị dịch vụ của khách hàng. Dạng nguồn lực xã hội tác động gián tiếp đến hành vi tham gia bằng cách làm gia tăng nguồn lực văn hóa và nguồn lực vật lý của khách hàng. Một số hàm ý quản trị cũng được thảo luận ở bài báo này.

\section{ABSTRACT}

Value is co-created and determined by customers. Customer operant resources are very important for service value co-creation and determination activities. Customer operant resources consist of 3 types - cultural, social and physical resources. Results of this empirical research indicate that in the service process, firms have the opportunity to affect customers' cultural and physical resources through communication effectiveness. These are also two types of customer operant resources that positively affect customers' service value co-creation participation behaviors The type of customer social operant resource indirectly affects customer participation behavior by increasing customers' cultural and physical resources. Some managerial implications are also discussed in this article. 


\section{Giới thiệu}

Sự thành công trong các hoạt động tạo sinh giá trị dịch vụ với KH (khách hàng) giúp DN (doanh nghiệp) đạt được các thành quả chiến lược như thị phần, doanh thu, ... (Gummesson \& Mele, 2010; Ostrom et al., 2010; Vargo \& Lusch, 2017). Tuy nhiên, giá trị dịch vụ phụ thuộc vào nguồn lực mà $\mathrm{KH}$ cung cấp trong quá trình cung cấp dịch vụ của $\mathrm{DN}$ (Grönroos, 2008; Vargo \& Lusch, 2017). Nghĩa là, nếu KH tích cực tham gia đóng góp nguồn lực của họ trong quá trình cung cấp dịch vụ của $\mathrm{DN}$ thì giá trị dịch vụ $\mathrm{KH}$ nhận được sẽ tốt hơn (Grönroos, 2011). Do đó, $\mathrm{DN}$ cần hỗ trợ các hành vi tham gia chủ động của $\mathrm{KH}$ với quá trình cung cấp dịch vụ của DN (Grönroos \& Gummerus, 2014; Vargo \& Lusch, 2004). Giao tiếp thông tin có hiệu quả với $\mathrm{KH}$ trong quá trình dịch vụ để nâng cao hiểu biết của $\mathrm{KH}$ là một phương thức hỗ trợ cho hành vi tham gia chủ động của KH (Auh, Bell, Mcleod, \& Shih, 2007; Grissemann \& Stokburger-Sauer, 2012). Nói cách khác, hiệu quả giao tiếp thông tin với KH giúp gia tăng nguồn lực tương tác (operant resources) của KH để họ tham gia tích cực vào quá trình dịch vụ (Arnould, Price, \& Malshe, 2006; Auh et al., 2007). Tuy nhiên, nguồn lực tương tác của KH gồm 3 dạng khác nhau, đó là nguồn lực vật lý (physical resources), nguồn lực văn hóa (cultural resources) và nguồn lực xã hội (social resources) (Arnould et al., 2006; Nguyen, 2018). Các nghiên cứu trước đây chỉ mới đề cập đến nguồn lực tương tác của KH một cách tổng quát, chưa cho thấy cụ thể dạng nguồn lực tương tác nào của $\mathrm{KH}$ sẽ được gia tăng thông qua hiệu quả giao tiếp thông tin của DN (Auh et al., 2007; Grissemann \& Stokburger-Sauer, 2012). Từ đó, câu hỏi thứ nhất được đặt ra là hiệu quả giao tiếp thông tin của $\mathrm{DN}$ với $\mathrm{KH}$ trong quá trình dịch vụ tác động ở mức độ nào đến mỗi một dạng nguồn lực tương tác của KH?. Hơn nữa, mức độ tác động của mỗi một dạng nguồn lực này đến hành vi tham gia của $\mathrm{KH}$ cũng chưa được khám phá trong các nghiên cứu trước đây (Nguyen, 2018; Nguyen, Pham, \& Pham, 2016). Do đó, câu hỏi thứ hai được đặt ra là mỗi dạng nguồn lực tương tác của $\mathrm{KH}$ tác động ở mức độ nào đến hành vi tham gia của $\mathrm{KH}$ trong quá trình dịch vụ? Tìm được câu trả lời cho các câu hỏi này không những đóng góp về mặt lý thuyết liên quan đến mối quan hệ giữa các dạng nguồn lực tương tác của $\mathrm{KH}$ với hành vi tham gia đồng tạo sinh giá trị của $\mathrm{KH}$ (Nguyen et al., 2016), mà còn giúp cho DN dịch vụ có được thông tin thuyết phục trong các quyết định chiến lược để nâng cao nguồn lực tương tác của $\mathrm{KH}$. Trên cơ sở đó, nghiên cứu này được hình thành với hai mục tiêu chính. Thứ nhất là, khám phá mức độ ảnh hưởng của hiệu quả giao tiếp thông tin của DN đối với mỗi một dạng nguồn lực tương tác của $\mathrm{KH}$. Thứ hai, mỗi một dạng nguồn lực tương tác của $\mathrm{KH}$ tác động ở mức độ nào đến hành vi tham gia của $\mathrm{KH}$ trong quá trình dịch vụ.

Nghiên cứu này được thực hiện với lĩnh vực y tế tại Việt Nam nhằm trả lời cho các câu hỏi nghiên cứu đã đề cập ở trên. Với những lĩnh vực có sự tương tác cao giữa $\mathrm{KH}$ và nhà cung cấp dịch vụ như y tế, giáo dục,... thì hành vi tham gia của $K H$ (e.g., bệnh nhân) trong quá trình dịch vụ là cần thiết để nhà cung cấp dịch vụ (e.g., bác sĩ, điều dưỡng,..) đưa ra giải pháp dịch vụ phù hợp (e.g., chẩn đoán, xét nghiệm,...) (Nguyen et al., 2016). Hơn nữa, ở những lĩnh vực này thì KH (e.g., bệnh nhân) thường có ít kiến thức hơn phía cung cấp dịch vụ (e.g., bác sĩ) (Nguyen et al., 2016). Do đó, hiệu quả giao tiếp thông tin của DN trong quá trình dịch vụ (e.g., khám chữa bệnh) có vai trò quan trọng để gia tăng nguồn lực tương tác của $\mathrm{KH}$ và hành vi tham gia của họ (e.g., kiến thức của bệnh nhân về bệnh lý).

Các phần tiếp theo sẽ lần lượt trình bày cơ sở lý thuyết và các giả thuyết nghiên cứu. Tiếp đến là về phương pháp nghiên cứu và kết quả thực nghiệm. Cuối cùng là phần thảo luận ý nghĩa lý thuyết, hàm ý thực tiễn và kết luận cho bài báo. 


\section{Cơ sở lý thuyết và mô hình nghiên cứu}

Để đạt được các mục tiêu đã đề cập ở trên, mục này trước tiên sẽ trình bày chi tiết các khái niệm nghiên cứu liên quan. Sau đó, mối quan hệ giữa các khái niệm và mô hình lý thuyết sẽ được trình bày.

\subsection{Các khái niệm}

Các khái niệm nghiên cứu được đề cập trong phần này bao gồm: Nguồn lực tương tác của $\mathrm{KH}$ (customer operant resources), Hiệu quả giao tiếp thông tin của DN (communication effectiveness), Hành vi tham gia của KH (customer participation behavior).

\section{Nguồn lụ̣c tương tác của Khách hàng (customer operant resources)}

Nguồn lực tương tác của KH mang tính cá nhân, phản ảnh năng lực cá nhân cùng với khả năng tương tác xã hội của cá nhân đó (Nguyen, 2018). Nguồn lực tương tác là các nguồn lực vô hình, không thể nhìn thấy được, mang bản chất động và không có giới hạn (Vargo \& Lusch, 2004). Điều này cũng có nghĩa rằng nguồn lực tương tác có thể thay đổi qua thời gian hoặc bối cảnh tình huống. Nguồn lực tương tác có khả năng tác động đến các nguồn lực khác để tạo ra một tác động (Vargo \& Lusch, 2004). Nguồn lực xã hội (social resource), nguồn lực vật lý (physical resource) và nguồn lực văn hóa (cultural resource) là 3 dạng nguồn lực tương tác của một cá nhân (e.g., bệnh nhân) (Arnould et al., 2006; Baron \& Warnaby, 2011). Các nội dung tiếp theo trình bày chi tiết về 3 dạng nguồn lực này.

Nguồn lục xã họi (social resource):

Nguồn lục xã hộ $i$ được định nghĩa là mạng lưới các mối quan hệ xã hội của KH mà qua đó họ tiếp cận nguồn lực ở các mức độ khác nhau. Bao gồm các mối quan hệ với các thành viên trong gia đình, bạn bè, đồng nghiệp và các mối quan hệ có tính thương mại khác (Arnould et al., 2006). Khi các cá nhân thiếu nguồn lực để đạt mục tiêu của mình thì họ có thể dựa vào các mối quan hệ xã hội để tiếp cận nguồn lực từ cá nhân khác (Nguyen, 2018). Với bối cảnh của nghiên cứu này, nguồn lực xã hội của bệnh nhân là sự hỗ trợ từ người khác, đặc biệt là các thành viên trong gia đình, về thông tin, sự cảm thông chia sẻ, sự khuyến khích hoặc các hỗ trợ khác cho quá trình khám và điều trị bệnh.

Nguồn lục văn hóa (cultural resource):

Nguồn lục văn hóa đề cập chủ yếu đến kiến thức chuyên môn của $\mathrm{KH}$ (Baron \& Warnaby, 2011). Với bối cảnh của nghiên cứu này, nguồn lực văn hóa của bệnh nhân đề cập đến kiến thức của bệnh nhân về bệnh lý và các hiểu biết cần thiết về thủ tục, qui trình khám và điều trị bệnh.

\section{Nguồn lục vật lý (physical resources):}

Nguồn lực vật lý đề cập đến độ mạnh về thể chất và tinh thần của một cá nhân. Bao gồm sự tự tin vào năng lực bản thân, cảm xúc và sự lạc quan (Arnould et al., 2006). Sự tự tin vào năng lực bản thân có thể thay đổi theo ngữ cảnh tình huống, có thể bị thay đổi bởi các yếu tố bên ngoài (Arnould et al., 2006; Nguyen, 2018). Trong khi sự lạc quan thể hiện tính cách cá nhân của một người, cảm xúc phụ thuộc sự tự tin vào năng lực của cá nhân (Bandura, 1977). Nếu cá nhân có sự tự tin vào năng lực thấp thì họ sẽ có cảm xúc tiêu cực, ngược lại sự tự tin vào năng lực là cao thì họ có cảm xúc tích cực để hành động. Nghiên cứu này được thực hiện với trọng tâm là ở quá trình dịch vụ với sự tương tác của $\mathrm{DN}$ và $\mathrm{KH}$, do đó sự tự tin vào năng lực được lựa chọn để đại diện cho nguồn lực vật lý của bệnh nhân. 


\section{Hiệu quả giao tiếp của DN với KH (communication effectiveness)}

Hiệu quả giao tiếp của DN với $\mathrm{KH}$ (communication effectiveness) đề cập đến việc chia sẻ chính thức hoặc không chính thức các thông tin có ý nghĩa và kịp thời giữa $\mathrm{DN}$ với $\mathrm{KH}$ theo cách đồng cảm với nhau (Sharma \& Patterson, 1999). Mục đích là để giáo dục hoặc thông báo cho KH thông tin cần thiết liên quan đến dịch vụ theo cách họ có thể hiểu được (Auh et al., 2007; Sharma \& Patterson, 1999).

Giao tiếp giữa DN với $\mathrm{KH}$ có thể được thực hiện với nhiều hình thức khác nhau như lời nói, chữ viết, cử chỉ, thái độ,...Với trọng tâm hướng đến hiệu quả giao tiếp trong quá trình dịch vụ với sự tương tác trực tiếp giữa $\mathrm{DN}$ với $\mathrm{KH}$, nghiên cứu này chỉ tập trung vào hiệu quả giao tiếp miệng bằng lời nói giữa nhân viên dịch vụ (e.g., bác sĩ, điều dưỡng) của $\mathrm{DN}$ (bệnh viện) với KH (bệnh nhân). Nghĩa là, với bối cảnh dịch vụ y tế của nghiên cứu này, hiệu quả giao tiếp trực tiếp đề cập đến quá trình nhân viên dịch vụ trực tiếp tiếp xúc KH như bác sĩ, điều dưỡng,... cung cấp thông tin có ý nghĩa và kịp thời cho bệnh nhân trong quá trình khám và điều trị bệnh theo cách mà bệnh nhân có thể hiểu rõ. Thông tin cung cấp có thể bao gồm giải thích rõ các triệu chứng của bệnh, qui trình, thủ tục khám/tái khám bệnh, thông tin các tác dụng phụ có thể có, các dặn dò (hướng dẫn) bệnh nhân trong lúc khám và sau khi khám bệnh như cách uống thuốc, chế độ ăn uống, tự chăm sóc bản thân,...

\section{Hành vi tham gia của khách hàng (customer participation behavior)}

Hành vi tham gia của $\mathrm{KH}$ vào quá trình dịch vụ được định nghĩa gồm những hành vi có liên quan đến việc tạo sinh và chuyển giao dịch vụ (Auh et al., 2007). Những hành vi này của KH được giới hạn trong phạm vi tương tác trực tiếp của quá trình dịch vụ (Grörnroos \& Voima, 2013; Nguyen, 2018). Theo Yi và Gong (2013), hành vi tham gia của KH là khái niệm bậc hai bao gồm 4 thành phần là: Tìm kiếm thông tin (information seeking), Chia sẻ thông tin (information sharing), Hành vi trách nhiệm (responsible behavior) và Tương tác cá nhân (personal interaction). Tìm kiếm thông tin đề cập đển những hành vi $\mathrm{KH}$ tìm kiếm các thông tin liên quan để làm rõ các yêu cầu của dịch vụ, thỏa mãn nhu cầu nhận thức của họ, tìm hiểu cách thức họ thực hiện các công việc trong quá trình dịch vụ. Bệnh nhân có thể tìm kiếm thông tin liên quan đến qui trình khám chữa bệnh, các phương pháp điều trị bệnh bản thân đang gặp phải và hiệu quả của chúng, ... Tuy nhiên, đây là hành vi diễn ra trước quá trình dịch vụ, do đó không được đề cập trong nghiên cứu này (Nguyen et al., 2016). Chia sẻ thông tin đề cập đến việc KH cung cấp thông tin cho nhân viên dịch vụ để họ thực hiện tốt công việc tạo sinh và chuyển giao dịch vụ. Chia sẻ thông tin là hành vi thể hiện sự đóng góp nguồn lực của $\mathrm{KH}$ vào việc tạo sinh và chuyển giao dịch vụ. Thông qua chia sẻ thông tin, $\mathrm{KH}$ giúp nhân viên dịch vụ chuyển giao dịch vụ đúng như nhu cầu của KH. Với nghiên cứu này, bệnh nhân có thể chia sẻ với nhân viên dịch vụ (bác sĩ, điều dưỡng, ...) thông tin về tình trạng bệnh, tiền sử bệnh, triệu chứng hiện tại, lịch sử điều trị, phương pháp điều trị mong muốn, hoàn cảnh kinh tế, gia đình, công việc, ... Hành vi trách nhiệm đề cập đến việc $\mathrm{KH}$ thực hiện nhiệm vụ của họ khi tương tác với nhà cung cấp dịch vụ trong quá trình dịch vụ (Ennew \& Binks, 1999; Yi \& Gong, 2013). Trong nghiên cứu này, hành vi trách nhiệm thể hiện qua sự hợp tác của bệnh nhân trong quá trình khám/chẩn đoán bệnh, tuân thủ các yêu cầu và hướng dẫn của bác sĩ/điều dưỡng,...Tương tác cá nhân đề cập đến mối quan hệ tương tác giữa $\mathrm{KH}$ và nhân viên, thể hiện qua sự thân thiện, lịch sự và tôn trọng lẫn nhau giữa $\mathrm{KH}$ với nhân viên dịch vụ. Trong lĩnh vực y tế, tương tác cá nhân của bệnh nhân là thái độ tôn trọng, lịch sự và thân thiện của bệnh nhân với nhân viên dịch vụ. Đây là nền tảng cho sự thành công của đồng tạo sinh giá trị dịch vụ (Ennew \& Binks, 1999). 


\subsection{Các giả thuyết và mô hình nghiên cứu}

Từ các cơ sở lý thuyết trên, mục này trình bày các giả thuyết về mối quan hệ giữa các khái niệm nghiên cứu như dưới đây.

\section{Hiệu quả giao tiếp thông tin của DN và Nguồn lục tương tác của KH}

Khách hàng là tác nhân tích hợp nguồn lực để tạo giá trị cho chính họ (Vargo \& Lusch, 2004). Trong đó, nguồn lực tương tác có vai trò quan trọng đối với hoạt động tích hợp nguồn lực của KH (Vargo \& Lusch, 2004). Giá trị dịch vụ khám chữa bệnh được tạo sinh và được xác định bởi bệnh nhân trên cơ sở kiến thức/thông tin của họ. Nói cách khác, chính $\mathrm{KH}$, chứ không phải nhà cung cấp dịch vụ (e.g., bệnh viện), là tác nhân tạo sinh và xác định giá trị dịch vụ thông qua hoạt động tích hợp các nguồn lực của họ (Vargo \& Lusch, 2004). Vì vậy, giá trị dịch vụ được xác định bởi $\mathrm{KH}$ phụ thuộc vào khả năng của $\mathrm{DN}$ trong việc cung cấp thêm nguồn lực (kiến thức/kỹ năng/thông tin) vào tổng các nguồn lực mà KH đang có.

Trong số các dạng nguồn lực của $\mathrm{KH}$, nguồn lực xã hội liên quan đến các mối quan hệ xã hội với các thành viên trong gia đình, bạn bè, ... (Arnould et al., 2006). Do đó, DN không thể tác động để gia tăng dạng nguồn lực xã hội của bệnh nhân trong quá trình dịch vụ. Ngược lại, trong các tương tác của quá trình dịch vụ, DN có cơ hội tác động để bổ sung nguồn lực văn hóa và nguồn lực vật lý cho bệnh nhân, qua đó ảnh hưởng giá trị dịch vụ được cảm nhận bởi bệnh nhân (Grönroos \& Voima, 2013). Vì vậy, nghiên cứu này chỉ xem xét đến tác động của Hiệu quả thông tin đến nguồn lực văn hóa và nguồn lực vật lý của bệnh nhân. Mỗi bệnh nhân khi đến khám và điều trị bệnh có những đặc trưng cá nhân không giống nhau như trình độ, sự hiểu biết về bệnh, mối quan hệ bạn bè, đồng nghiệp, trải nghiệm dịch vụ,...Nói cách khác, nguồn lực tương tác của bệnh nhân là không giống nhau và có thể có trường hợp bệnh nhân chưa có đầy đủ thông tin/kiến thức để phối hợp khám và điều trị bệnh thành công với bệnh viện. Trong các tương tác trực tiếp với bệnh nhân, nhân viên tiếp xúc KH (e.g., bác sĩ, điều dưỡng,...) cung cấp và giải thích thông tin cần thiết, phù hợp với đặc thù của từng bệnh nhân theo cách đồng cảm với họ thì kiến thức/kỹ năng và sự tự tin của bệnh nhân sẽ được cải thiện. Nghĩa là, hiệu quả giao tiếp thông tin của $\mathrm{DN}$ trong quá trình dịch vụ gia tăng nguồn lực văn hóa và nguồn lực vật lý của $\mathrm{KH}$. Trên cơ sở các lập luận trên, giả thuyết được đề xuất như sau:

Hla: Hiệu quả giao tiếp thông tin của bệnh viện trong quá trình dịch vu tác động tích cưc đến Nguồn lực văn hóa của bệnh nhân

H1b: Hiệu quả giao tiếp thông tin của bệnh viện trong quá trình dịch vụ tác động tích cực đến Nguồn lực vật lý của bệnh nhân

\section{Nguồn lục tương tác của bệnh nhân và Hành vi tham gia đồng tạo sinh giá trị}

Nguồn lực văn hóa thể hiện kỹ năng và kiến thức chuyên môn của bệnh nhân về bệnh, qui trình, thủ tục khám và điều trị bệnh. Kỹ năng và kiến thức này có thể được tích lũy thông qua quá trình học tập và trải nghiệm của bệnh nhân. Trong các tương tác với nhân viên dịch vụ, kỹ năng và kiến thức này của bệnh nhân rất cần thiết cho việc trao đổi thông tin giữa bệnh nhân và nhân viên để chẩn đoán và điều trị bệnh chính xác. Bệnh nhân càng hiểu biết nhiều về triệu chứng bệnh lý, tiền sử của bệnh thì càng có xu hướng chia sẻ thông tin với nhân viên. Hơn nữa, khi một bệnh nhân có kiến thức về bệnh thì khoảng cách tri thức giữa hai phía tham gia (e.g., bệnh nhân và bác sĩ/điều dưỡng) trong tương tác sẽ ít hơn, đây là tiền đề để bệnh nhân tham gia trao đổi hiệu quả với nhân viên dịch vụ. Dựa vào các cơ sở trên, giả thuyết được đề xuất là: 
H2a: Nguồn lực văn hóa tác động tích cưc đến Hành vi tham gia của bệnh nhân trong quá trình dịch vu

Bên cạnh nguồn lực văn hóa, nguồn lực xã hội cung cấp cho bệnh nhân sự hỗ trợ ở các khía cạnh khác nhau từ gia đình, bạn bè, đồng nghiệp, thậm chí là từ các bệnh nhân khác. Thông tin, kiến thức mà bệnh nhân có được từ mạng lưới xã hội giúp bệnh nhân hiểu hơn về bệnh lý của bản thân, cũng như quá trình dịch vụ của bệnh viện. Hơn nữa, các thành viên gia đình có thể đóng vai trò như là người đi theo hỗ trợ hoặc dặn dò, chỉ bảo bệnh nhân trong quá trình dịch vụ. Từ đó, tạo thuận lợi cho hành tham gia chủ động của bệnh nhân trong quá trình khám và điều trị bệnh. Dựa vào cơ sở trên, giả thuyết được đề xuất là:

H2b: Nguồn lực xã hội tác động tích cực đến Hành vi tham gia của bệnh nhân trong quá trình dịch vu

Ngoài nguồn lực văn hóa và nguồn lực xã hội, nguồn lực vật lý là dạng nguồn lực thứ 3 trong nguồn lực tương tác của cá nhân (Arnould et al., 2006; Nguyen, 2018). Nguồn lực vật lý của cá nhân có thể được nhận diện thông qua nhận thức của cá nhân về năng lực để thực hiện một hành động cụ thể nào đó (Bandura, 1977). Nghĩa là, nguồn lực vật lý (năng lực bản thân) thể hiện nhận thức về mức độ kiểm soát một hành động, gồm cả động lực và nỗ lực để thực hiện hành động đó của một cá nhân (Nguyen, 2018). Theo lý thuyết nhận thức xã hội (Social Cognitive Theory), nguồn lực vật lý ảnh hưởng đến khả năng một cá nhân khởi xướng một hành vi nhất định và sau đó nỗ lực bền bỉ để thực hiện hành vi đó dù có gặp trở ngại hoặc khó khăn (Bandura, 1977; Nguyen, 2018). Do đó, với bối cảnh nghiên cứu này, nguồn lực vật lý của bệnh nhân ở mức độ cao có vai trò quan trọng trong việc kích thích hành vi tham gia đồng tạo sinh của họ trong quá trình dịch vụ. Do đó, giả thuyết được đề xuất:

H2c: Nguồn lực vật lý tác động tích cực đến Hành vi tham gia của bệnh nhân trong quá trình dịch $v u$

\section{Đóng góp của nguồn lực xã hội vào tổng Nguồn lực tương tác của bệnh nhân}

Một cách tổng quát, theo Hobfoll, Halbesleben, Neveu, và Westman (2018) khi các cá nhân thiếu nguồn lực để đạt được mục đích của mình, họ có xu hướng dựa vào các mối quan hệ xã hội để tiếp cận các nguồn lực họ cần.

Với bối cảnh của nghiên cứu này, như đã đề cập, nguồn lực xã hội cung cấp cho bệnh nhân sự hỗ trợ từ những người khác, đặc biệt là các thành viên gia đình về thông tin liên quan đến bệnh lý và quá trình dịch vụ của bệnh viện, sự cảm thông, sự động viên và ủng hộ (McCollKennedy et al., 2017). Nghĩa là, bệnh nhân với mức độ cao của nguồn lực xã hội được kỳ vọng sẽ có nhiều thông tin, kiến thức về bệnh lý cũng như quá trình dịch vụ. Nói cách khác, nguồn lực văn hóa có xu hướng tăng cao theo sự gia tăng về nguồn lực xã hội của bệnh nhân. Do đó, giả thuyết được đề xuất là:

\section{H3a: Nguồn lực xã hội tác động tích cực đến nguồn lực văn hóa của bệnh nhân}

Hơn nữa, sự cảm thông, động viên, ủng hộ và mối quan hệ chặt chẽ với các cá nhân khác trong các mối quan hệ xã hội, đặc biệt là các thành viên trong gia đình có thể giúp bệnh nhân giải tỏa căng thẳng, khuyến khích các cảm xúc tích cực, và gia tăng sự tự tin vào năng lực bản thân (Nguyen, 2018). Điều này có nghĩa là, nguồn lực xã hội tác động tích cực đến nguồn lực vật lý của bệnh nhân. Từ cơ sở trên, giả thuyết được đề xuất là:

H3b: Nguồn lục xã hội tác động đến nguồn lục vật lý của bệnh nhân 
Hình 1.

Trên cơ sở các biện luận và giả thuyết ở trên, mô hình nghiên cứu được trình bày như ở

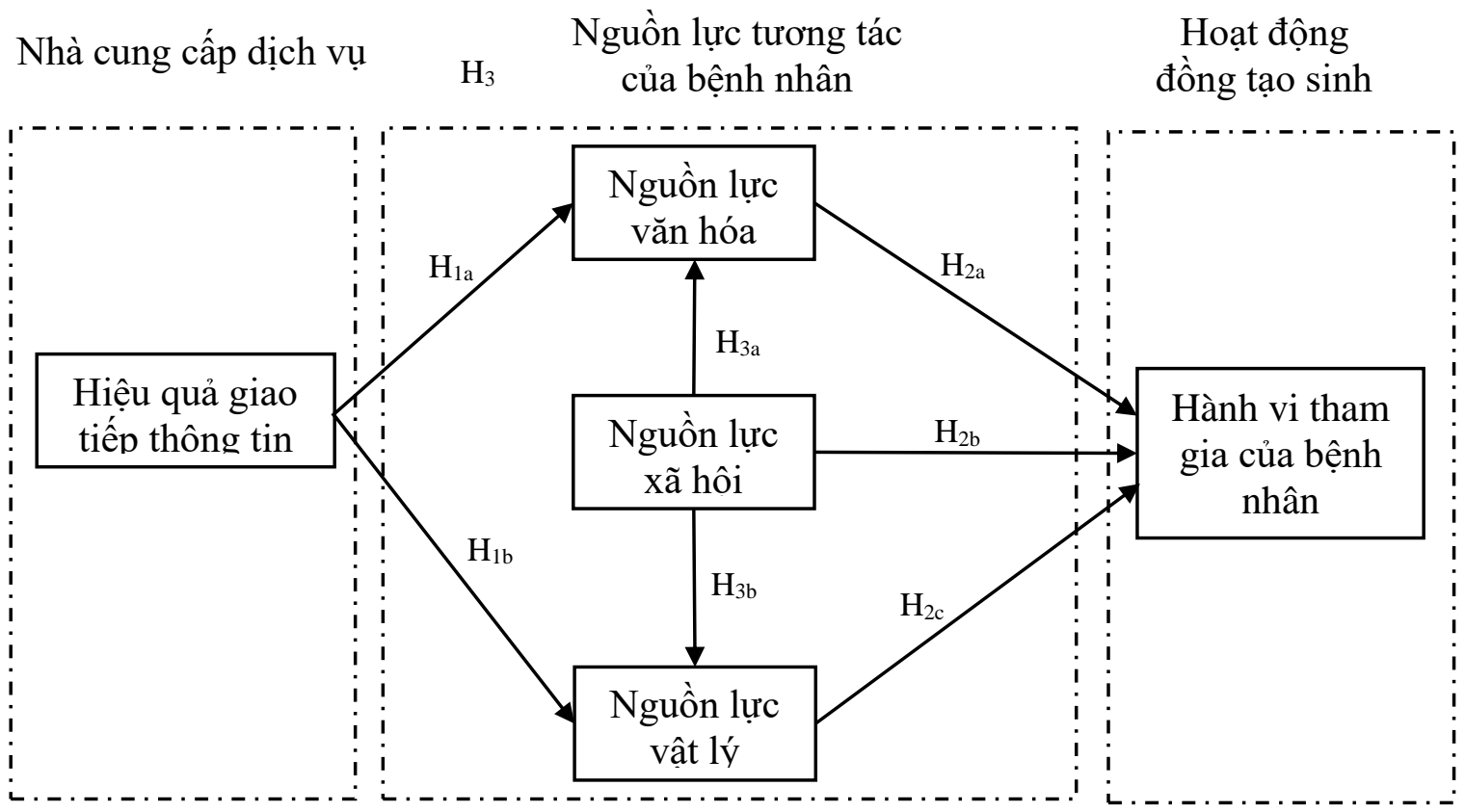

Hình 1. Mô hình nghiên cứu

\section{Phương pháp nghiên cứu}

Nghiên cứu sử dụng phương pháp định lượng để kiểm định mô hình và các giả thuyết. Dữ liệu được thu thập qua một cuộc khảo sát bệnh nhân tại các bệnh viện ở Thành phố Hồ Chí Minh (TP. HCM). Đối tượng khảo sát là bệnh nhân bị bệnh đái tháo đường mãn tính.

Công cụ thu thập dữ liệu là bảng câu hỏi có cấu trúc dùng thang đo Likert 5 điểm. Mẫu được chọn theo phương pháp thuận tiện, đối tượng khảo sát được tiếp cận ở khâu cuối cùng của quá trình dịch vụ, cụ thể là bệnh nhân đang đợi để lấy/mua thuốc từ quầy thuốc của bệnh viện. Bệnh nhân được đề nghị nghĩ về đợt thăm khám vừa trải qua và thể hiện mức độ đồng ý với các phát biểu trong bảng câu hỏi.

Các thang đo được kế thừa từ các nghiên cứu trước, có hiệu chỉnh (thông qua bước nghiên cứu định tính sơ bộ) cho phù hợp với bối cảnh nghiên cứu (xem Bảng 2 ). Cụ thể, thang đo Hiệu quả giao tiếp thông tin được kế thừa từ Sharma và Patterson (1999) với 4 biến quan sát. Hành vi tham gia của bệnh nhân với 3 thành phần được đo lường bằng 11 biến quan sát, kế thừa từ Yi và Gong (2013). Thang đo nguồn lực tương tác gồm nguồn lực văn hóa (4 biến quan sát), nguồn lực vật lý ( 3 biến quan sát) và nguồn lực xã hội (3 biến quan sát) được kế thừa từ Nguyen (2018). Phương pháp phân tích nhân tố khám phá (EFA), khẳng định (CFA) và mô hình cấu trúc tuyến tính SEM với sự hỗ trợ của phần mềm AMOS được sử dụng để phân tích dữ liệu.

\section{Phân tích kết quả}

\section{1. Đặc điểm mẫu}

Mẫu gồm 202 bệnh nhân được thu thập từ 7 bệnh viện trên địa bàn TP. HCM, đáp ứng 
yêu cầu về cỡ mẫu tối thiểu (là 150) cho các nghiên cứu dạng kiểm định thống kê như nghiên cứu này (Hair, Black, Babin, \& Anderson, 2010). Đặc trưng của mẫu theo giới tính, nghề nghiệp, nhóm tuổi, thu nhập được trình bày ở Bảng 1 . Theo đó, phân bố mẫu theo nghề nghiệp với $46 \%$ công nhân viên, $39,1 \%$ nội trợ/hưu trí, $14,1 \%$ làm nghề tự do/nông dân. Theo giới tính có $57,4 \%$ là nam giới, $42,6 \%$ là nữ giới. Nhóm tuổi chiếm tî̉ lệ cao trong mẫu là trên 50 tuổi với $45 \%$, từ 41 đến 50 là $29,2 \%$, từ 30 đến 40 là $19,3 \%$ và dưới 30 tuổi chiếm $6,4 \%$. Phân bố mẫu theo thu nhập, dưới 5 triệu đồng chiếm tỉ lệ cao nhất với 35,1\%. Các đặc trưng này cho thấy sự đa dạng của mẫu nghiên cứu và phù hợp cho kiểm định thống kê.

\section{Bảng 1}

Đặc trưng của mẫu nghiên cứu

\begin{tabular}{|c|c|c|c|c|c|}
\hline Yếu tố & Tần suất & $\%$ & Yếu tố & Tần suất & $\%$ \\
\hline \multicolumn{3}{|l|}{ Nghề nghiệp: } & \multicolumn{3}{|l|}{ Giới tính: } \\
\hline - Công nhân viên & 93 & 46.0 & - Nữ & 86 & 42,6 \\
\hline - Nội trợ/hưu trí & 79 & 39.1 & - Nam & 116 & 57,4 \\
\hline - Nông dân/tự do & 30 & 14.9 & \multicolumn{3}{|c|}{ Thu nhập (triệu đồng): } \\
\hline \multicolumn{3}{|l|}{ Nhóm tuổi: } & - Dưới 5 triệu & 71 & 35,1 \\
\hline - Dưới 30 & 13 & 6,4 & - Từ 5 tỷ đến 8 & 43 & 21,3 \\
\hline - Từ 30 đến 40 & 39 & 19,3 & - Từ 9 tỷ đến 15 & 40 & 19,8 \\
\hline - Từ 41 đến 50 & 59 & 29,2 & - Trên 15 & 48 & 23,8 \\
\hline - Trên 50 & 91 & 45,0 & & & \\
\hline
\end{tabular}

Nguồn: Kết quả phân tích dữ liệu của nhóm nghiên cứu

\subsection{Kiểm định mô hình thang đo}

Phân tích nhân tố khám phá EFA được thực hiện để đánh giá cấu trúc thang đo cho các khái niệm nghiên cứu. Kết quả EFA cho thấy thang đo các khái niệm đảm bảo cấu trúc đơn hướng khi các biến quan sát đều có hệ số tải cao $(>0,5)$ ở nhân tố liên quan (Hair et al., 2010). Tiếp theo, phương pháp phân tích CFA được sử dụng để đánh giá mô hình đo lường (Arbuckle \& Wothke, 1999). Có 03 biến quan sát bị loại vì có tương quan cao giữa các sai số. Kết quả CFA còn lại 22 biến quan sát đo lường 7 khái niệm nghiên cứu có các chỉ số phù hợp mô hình đạt yêu cầu. Cụ thể, mô hình đo lường có các chỉ số phù hợp như sau: Chi-square $\mathrm{x}^{2}(\mathrm{df}=188)=331,018 ; \quad \mathrm{p}=0,00 ; \quad$ Chi-square $/ \mathrm{df}=1,761 ; \quad \mathrm{GFI}=0,90 ; \quad \mathrm{TLI}=0,90 ; \quad \mathrm{CFI}=0,915 ;$ RMSEA= 0,062 (Hair et al., 2010). Như vậy, mô hình đo lường phù hợp với dữ liệu thực tế. Bảng 2 trình bày hệ số tải nhân tố, độ tin cậy tổng hợp $(\mathrm{CR})$ và tổng phương sai trích (AVE) của các thang đo. Các hệ số tải nhân tố dao động từ 0,66 đến 0,90 . Phương sai (AVE) của các thang đo dao động từ 0,50 đến 0,583 . Độ tin cậy tổng hợp $(\mathrm{CR})$ của 7 thang đo dao động từ 0,749 đến 0,820 . Hệ số tương quan giữa các khái niệm dao động từ 0,04 đến 0,68 , nhỏ hơn căn bậc hai của AVE tương ứng (Fornell \& Larcker, 1981). Do đó, các thang đo đạt yêu cầu về độ tin cậy, độ giá trị hội tụ và giá trị phân biệt. 


\section{Bảng 2}

Kết quả phân tích CFA các thang đo

\begin{tabular}{|c|c|}
\hline Tóm tắt nội dung biến quan sát & $\begin{array}{l}\text { Hệ số tải } \\
\text { chuấn hóa }\end{array}$ \\
\hline \multicolumn{2}{|l|}{ Hiệu quả giao tiếp thông tin $(\mathrm{CR}=0,820 ; \mathrm{AVE}=0,533)$ - Nhân viên $\mathrm{BV} \ldots$} \\
\hline cung cấp đầy đủ thông tin để giúp nâng cao hiểu biết về bệnh & 0,77 \\
\hline $\begin{array}{l}\text { giải thích rõ ràng những vấn đề cần thiết (thủ tục, phương pháp điều trị...) khi đến } \\
\text { khám và điều trị }\end{array}$ & 0,73 \\
\hline giải thích rõ những thuận lợi và khó khăn khi khám và điều trị bệnh & 0,70 \\
\hline cung cấp đầy đủ những thông tin có giá trị với tôi & 0,71 \\
\hline \multicolumn{2}{|l|}{ Nguồn lực vật lý của KH (CR= 0,767; AVE=0,525 ) - Tôi... } \\
\hline có thể giải quyết tốt các vấn đề sức khỏe của bản thân nếu cố gắng hết sức & 0,65 \\
\hline có thể đưa ra cách xử lý phù hợp cho bản thân khi gặp vấn đề về sức khỏe & 0,81 \\
\hline giữ được bình tĩnh khi gặp vấn đề về sức khỏe vì tin vào khả năng của mình & 0,71 \\
\hline \multicolumn{2}{|l|}{ Nguồn lụcc văn hóa của KH (CR= 0,805; AVE= 0,583 ) - Tôi... } \\
\hline biết rõ qui trình khám và điều trị bệnh tại $\mathrm{BV}$ & Loại \\
\hline biết các bước cần làm trong quá trình khám và chữa bệnh tại $\mathrm{BV}$ & 0,90 \\
\hline biết cách hợp tác với bác sĩ và nhân viên của $\mathrm{BV}$ nhằm mang lại lợi ích tốt nhất & 0,69 \\
\hline dễ dàng hiểu được các hướng dẫn trong quá trình khám và điều trị tại BV & 0,69 \\
\hline \multicolumn{2}{|l|}{ Nguồn lực xã hội của KH (CR=0,749; AVE= 0,506 ) - Tôi... } \\
\hline nhận được nhiều hỗ trợ hữu ích từ người thân và bạn bè trong quá trình điều trị bệnh & 0,69 \\
\hline được người thân, bạn bè, đồng nghiệp chia sẻ nhiều thông tin về điều trị bệnh tạiBV & 0,74 \\
\hline biết hầu hết các thông tin về $\mathrm{BV}$ từ diễn đàn/cộng đồng mạng/internet/trang web của $\mathrm{X}$ & 0,70 \\
\hline \multicolumn{2}{|l|}{ Hành vi chia sẻ thông tin của $\mathrm{KH}(\mathrm{CR}=0,771 ; \mathrm{AVE}=0,532)$ - Tôi... } \\
\hline $\begin{array}{l}\text { thường chia sẻ mong muốn về kết quả điều trị cho nhân viên ở X khi đến khám và điều } \\
\text { trị ở đây }\end{array}$ & Loại \\
\hline luôn kể hết diễn tiến bệnh của mình cho nhân viên ở X khi đến khám và điều trị ở đây & 0,77 \\
\hline thường kể rõ những thông tin mà bản thân nghĩ là cần thiết cho nhân viên ở X & 0,80 \\
\hline không giấu giếm bất kỳ thông tin nào liên quan đến bệnh khi được nhân viên ở X hỏi & 0,60 \\
\hline \multicolumn{2}{|l|}{ Hành vi trách nhiệm của KH $(\mathrm{CR}=0,761 ; \mathrm{AVE}=0,50)$ - Tôi... } \\
\hline $\begin{array}{l}\text { luôn ghi nhớ và tuân theo lời dặn của nhân viên ở } X \text { trong điều trị và tự kiểm soát bệnh } \\
\text { hàng ngày }\end{array}$ & 0,66 \\
\hline tái khám theo đúng lịch hẹn của nhân viên ở X & 0,72 \\
\hline luôn tuân thủ tốt việc tự kiểm soát bệnh hiệu quả theo hướng dẫn của nhân viên ở $\mathrm{X}$ & 0,75 \\
\hline \multicolumn{2}{|l|}{ Hành vi tương tác cá nhân của $K H(C R=0,774 ; A V E=0,535)$ - Tôi... } \\
\hline luôn thân thiện với nhân viên ở $\mathrm{X}$ & 0,80 \\
\hline có thái độ dễ chịu với nhân viên ở X ngay cả khi không vừa ý & 0,72 \\
\hline luôn lịch sự với nhân viên ở X & Loại \\
\hline không thô lỗ với nhân viên ở X & 0,66 \\
\hline
\end{tabular}

Nguồn: Kết quả phân tích dữ liệu của nhóm nghiên cứu 


\subsection{Kiểm định các giả thuyết với mô hình cấu trúc SEM}

Mô hình cấu trúc SEM với phương pháp maximum likelihook (ML) được ước lượng để kiểm định các giả thuyết nghiên cứu. Kết quả ước lượng cho thấy mức độ phù hợp tốt của mô hình với dữ liệu thực tế, các chỉ số đạt như sau: Chi-square $\mathrm{x}^{2}(\mathrm{df}=199)=346,432 ; \mathrm{p}=0,00$; Chisquare/df $=1,741 ; \mathrm{GFI}=0,90 ; \mathrm{TLI}=0,90 ; \mathrm{CFI}=0,908 ; \mathrm{RMSEA}=0,061$.

Kết quả ở Bảng 3 cho thấy ngoại trừ giả thuyết $\mathrm{H}_{2 b}$ không được ủng hộ (p-value $=0,62$ $>0,05)$, các giả thuyết còn lại đều được ủng hộ. Theo đó, giả thuyết $\mathrm{H}_{1 \mathrm{a}}$ thể hiện quan hệ giữa Hiệu quả giao tiếp thông tin với nguồn lực văn hóa của bệnh nhân có $\beta=0,54(\mathrm{p}=0,000)$; giả thuyết $\mathrm{H}_{1 \mathrm{~b}}$ thể hiện quan hệ giữa Hiệu quả giao tiếp thông tin với nguồn lực vật lý của bệnh nhân có $\beta=0,20(\mathrm{p}=0,010)$; giả thuyết $\mathrm{H}_{2 \mathrm{a}}$ thể hiện quan hệ giữa Nguồn lực văn hóa và Hành vi tham gia của bệnh nhân có $\beta=0,670(\mathrm{p}=0,000)$; giả thuyết $\mathrm{H}_{2 \mathrm{c}}$ thể hiện mối quan hệ giữa Nguồn lực vật lý với Hành vi tham gia có $\beta=0,36(\mathrm{p}=0,000)$; giả thuyết $\mathrm{H}_{3 \mathrm{a}}$ thể hiện quan hệ giữa Nguồn lực xã hội và nguồn lực văn hóa của bệnh nhân có $\beta=0,20(\mathrm{p}=0,010)$; giả thuyết $\mathrm{H}_{3 b}$ thể hiện quan hệ giữa nguồn lực xã hội với nguồn lực vật lý của bệnh nhân có $\beta=0,21$ (p $=0,010)$. Tóm lại, có 6 trên 7 giả thuyết được ủng hộ trong nghiên cứu này.

\section{Bảng 3}

Kết quả kiểm định giả thuyết

\begin{tabular}{|c|l|c|c|c|}
\hline $\begin{array}{c}\text { Giả } \\
\text { thuyết }\end{array}$ & \multicolumn{1}{|c|}{ Mối quan hệ nhân quả } & $\begin{array}{c}\text { Hệ số } \\
\boldsymbol{\beta}\end{array}$ & P-value & Kết quả \\
\hline $\mathrm{H}_{1 \mathrm{a}}$ & Hiệu quả giao tiếp thông tin $\rightarrow$ Nguồn lực văn hóa & 0,54 & 0,00 & Ung hộ \\
\hline $\mathrm{H}_{1 \mathrm{~b}}$ & Hiệu quả giao tiếp thông tin $\rightarrow$ Nguồn lực vật lý & 0,20 & 0,01 & Ủng hộ \\
\hline $\mathrm{H}_{2 \mathrm{a}}$ & Nguồn lực văn hóa $\rightarrow$ Hành vi tham gia của bệnh nhân & 0,67 & 0,00 & Ủng hộ \\
\hline $\mathrm{H}_{2 \mathrm{~b}}$ & Nguồn lực xã hội $\rightarrow$ Hành vi tham gia của bệnh nhân & 0,04 & 0,62 & $\begin{array}{c}\text { Không } \\
\text { ủng hộ }\end{array}$ \\
\hline $\mathrm{H}_{2 \mathrm{c}}$ & Nguồn lực vật lý $\rightarrow$ Hành vi tham gia của bệnh nhân & 0,36 & 0,00 & Ủng hộ \\
\hline $\mathrm{H}_{3 \mathrm{a}}$ & Nguồn lực xã hội $\rightarrow$ Nguồn lực văn hóa & 0.20 & 0.01 & Ung hộ \\
\hline $\mathrm{H}_{3 \mathrm{~b}}$ & Nguồn lực xã hội $\rightarrow$ Nguồn lực vật lý & 0.21 & 0.01 & Ung hộ \\
\hline
\end{tabular}

Nguồn: Kết quả xử lý từ dữ liệu điều tra

\subsection{Thảo luận kết quả}

Nguồn lực tương tác của KH có vai trò quan trọng đối với giá trị dịch vụ được đồng tạo sinh với DN (Auh et al., 2007; Nguyen, 2018). Tìm hiểu dạng nguồn lực tương tác nào của $\mathrm{KH}$ bị tác động bởi hiệu quả giao tiếp thông tin của $\mathrm{DN}$ trong quá trình dịch vụ là mục đích hướng đến đầu tiên của nghiên cứu này. Kết quả nghiên cứu cho thấy, trong quá trình dịch vụ, giao tiếp thông tin hiệu quả của $\mathrm{DN}$ với $\mathrm{KH}$ có vai trò quan trọng đối với Nguồn lực văn hóa và Nguồn lực vật lý của KH. Nói cách khác, giao tiếp thông tin hiệu quả của DN với KH trong quá trình dịch vụ giúp gia tăng kiến thức và kỹ năng của KH (Nguồn lực văn hóa), đồng thời cũng giúp tăng tự tin vào năng lực bản thân (Nguồn lực vật lý) của KH. Điều này cung cấp thêm bằng chứng thực nghiệm về việc $\mathrm{DN}$ có thể ảnh hưởng đến giá trị dịch vụ đồng tạo sinh thông qua tương tác hiệu quả trong quá trình dịch vụ (Grönroos, 2017). Nghĩa là, DN cần hỗ trợ tạo sinh giá trị tốt nhất cho KH bằng cách chú trọng hiệu quả trong các giao tiếp tương tác thông tin với $\mathrm{KH}$. Về mức độ tác động, hiệu quả giao tiếp thông tin tác động nhiều hơn đến 
dạng Nguồn lực văn hóa của $K H(\beta=0,54)$ so với Nguồn lực vật lý $(\beta=0,20)$. Điều này có nghĩa rằng, kiến thức và kỹ năng chuyên môn của $\mathrm{KH}$ (e.g., bệnh nhân) được cải thiện đáng kể (so với sự tự tin vào năng lực) trong quá trình tương tác dịch vụ nếu như giao tiếp giữa $\mathrm{DN}$ và $\mathrm{KH}$ trong quá trình này có hiệu quả.

Nghiên cứu này cũng hướng đến mục đích thứ hai là tìm hiểu mức độ tác động của mỗi dạng nguồn lực tương tác khác nhau của KH (bao gồm Nguồn lực văn hóa, Nguồn lực xã hội, Nguồn lực vật lý) đến hành vi tham gia đồng tạo sinh giá trị của KH (e.g., bệnh nhân). Kết quả nghiên cứu chỉ ra rằng Nguồn lực văn hóa và Nguồn lực vật lý của bệnh nhân tác động trực tiếp đển hành vi tham gia trong quá trình dịch vụ. Nghĩa là khi bệnh nhân có mức độ kiến thức/thông tin càng nhiều và tự tin vào năng lực bản thân cao sẽ dễ dàng trao đổi, thảo luận với nhân viên dịch vụ trong quá trình tương tác dịch vụ. Kết quả nghiên cứu cũng cho thấy mức độ tác động đến hành vi tham gia trong quá trình dịch vụ của Nguồn lực văn hóa $(\beta=0,67)$ cao hơn nguồn lực vật lý $(\beta=0,36)$.

Kết quả nghiên cứu chỉ ra Nguồn lực xã hội không tác động trực tiếp nhưng có vai trò tác động gián tiếp đến hành vi tham gia của bệnh nhân trong quá trình dịch vụ. Nghĩa là, bệnh nhân với Nguồn lực xã hội cao sẽ làm gia tăng Nguồn lực văn hóa và Nguồn lực vật lý, từ đó tác động đến hành vi tham gia trong quá trình dịch vụ. Cụ thể hơn, mức độ tác động để đóng góp làm gia tăng tổng nguồn lực tương tác của $\mathrm{KH}$ thông qua Nguồn lực văn hóa $(\beta=0,20)$ và Nguồn lực vật lý $(\beta=0,21)$ của Nguồn lực xã hội là gần như nhau. Đây là một kết quả thực nghiệm quan trọng liên quan đến cấu trúc tạo thành nguồn lực tương tác tổng thể của $\mathrm{KH}$ (Arnould et al., 2006; Heinonen \& Strandvik, 2015; Nguyen, 2018).

\section{Kết luận và hàm ý}

Nghiên cứu đã dẫn đến một số điểm có ý nghĩa liên quan đến nguồn lực tương tác của $\mathrm{KH}$ trong quá trình dịch vụ. $\mathrm{KH}$ là tác nhân xác định giá trị dịch vụ bằng cách sử dụng nguồn lực tương tác (Vargo \& Lusch, 2008). Nguồn lực tương tác được cấu thành từ Nguồn lực văn hóa, Nguồn lực vật lý và Nguồn lực xã hội (Arnould et al., 2006). Nghiên cứu cho thấy dạng Nguồn lực văn hóa và Nguồn lực vật lý của KH chịu tác động của hiệu quả giao tiếp thông tin của $\mathrm{DN}$ trong quá trình dịch vụ. Do đó, trong quá trình dịch vụ $\mathrm{DN}$ có cơ hội cải thiện tổng nguồn lực tương tác của $\mathrm{KH}$ thông qua giao tiếp thông tin hiệu quả để tăng Nguồn lực văn hóa và Nguồn lực vật lý. Kết quả nghiên cứu cũng chỉ ra rằng Nguồn lực văn hóa và Nguồn lực vật lý tác động tích cực trực tiếp đến hành vi tham gia đồng tạo sinh giá trị trong quá trình dịch vụ của bệnh nhân. Nghĩa là, $\mathrm{DN}$ có thể hỗ trợ hoạt động đồng tạo sinh giá trị của KH (e.g., bệnh nhân) thông qua giao tiếp thông tin hiệu quả trong quá trình dịch vụ. Để làm được điều này, bệnh viện cần liên tục truyền thông nội bộ đến đội ngũ nhân viên dịch vụ (bác sĩ, điều dưỡng,...) về tầm quan trọng của việc cung cấp thông tin đầy đủ, giải thích rõ ràng và cảm thông với bệnh nhân trong các tương tác dịch vụ. Hơn nữa, trong các tương tác với $\mathrm{KH}$, bên cạnh các thông tin/kiến thức chuyên môn về bệnh lý, thủ tục, qui trình khám và điều trị, cần trao đổi và động viên bệnh nhân nhằm nâng cao sự tự tin của bệnh nhân với quá trình điều trị bệnh lý của bản thân.

Nghiên cứu đã đạt được các kết quả tích cực, tuy nhiên vẫn còn một số hạn chế như sau. Thứ nhất, nghiên cứu chỉ thực hiện với khách hàng (bệnh nhân) của dịch vụ y tế. Các nghiên cứu tiếp theo có thể khám phá thêm ở các lĩnh vực dịch vụ có sự tương tác cao giữa khách hàng và $\mathrm{DN}$ như giáo dục, bảo hiểm, ngân hàng. Thứ hai, mẫu nghiên cứu được lựa chọn thuận tiện với bệnh nhân bị đái tháo đường ở một số bệnh viện trên địa bàn TP.HCM. Nghiên cứu tiếp 
theo có thể khám phá với bệnh nhân bị các bệnh lý mãn tính khác như tim mạch, trĩ, rối loạn mỡ máu,... Thứ ba, nghiên cứu tiếp theo cũng có thể khám phá thêm lợi ích cảm nhận bởi khách hàng như là hậu tố của hành vi tham gia đồng tạo sinh với quá trình dịch vụ của $\mathrm{DN}$.

\section{LÒ̀I CÁM ƠN}

Nghiên cứu này được tài trợ bởi Trường Đại học Bách Khoa - ĐHQG-HCM trong khuôn khổ đề tài mã số T-QLCN-2018-59.

\section{Tài liệu tham khảo}

Arbuckle, J. L., \& Wothke, W. (1999). Amos 4.0 user's guide. Chicago, IL: SmallWaters Corporation.

Arnould, E. J., Price, L. L., \& Malshe, A. (2006). Toward a cultural resource-based theory of the customer. In R. F. Lusch \& S. L. Vargo (Eds.), The Service-dominant logic of marketing: Dialog, debate, and directions (pp. 91-104). Armonk, NY: ME Sharpe.

Auh, S., Bell, S. J., Mcleod, C., \& Shih, E. (2007). Co-production and customer loyalty in financial services. Journal of Retailing, 83(3), 359-370.

Bandura, A. (1977). Self-efficacy: Toward a unifying theory of behavioral change. Psychological Review, 84(2), 191-215.

Baron, S., \& Warnaby, G. (2011). Individual customers' use and integration of resources: Empirical findings and organizational implications in the context of value co-creation. Industrial Marketing Management, 40(2), 211-218.

Ennew, C. T., \& Binks, M. R. (1999). Impact of participative service relationships on quality, satisfaction and retention: An exploratory study. Journal of Business Research, 46(2), 121-132.

Fornell, C., \& Larcker, D. F. (1981). Evaluating structural equation models with unobservable variables and measurement error. Journal of Marketing Research, 18(1), 39-50.

Grissemann, U. S., \& Stokburger-Sauer, N. E. (2012). Customer co-creation of travel services: The role of company support and customer satisfaction with the co-creation performance. Tourism Management, 33(6), 1483-1492.

Grönroos, C. (2008). Service logic revisited: Who creates value? And who co-creates? European Business Review, 20(4), 298-314.

Grönroos, C. (2011). Value co-creation in service logic: A critical analysis. Marketing Theory, 11(3), 279-301.

Grönroos, C. (2017). On value and value creation in service: A management perspective. Journal of Creating Value, 3(2), 125-141.

Grönroos, C., \& Gummerus, J. (2014). The service revolution and its marketing implications: Service logic vs service-dominant logic. Managing Service Quality, 24(3), 206-229.

Grönroos, C., \& Voima, P. (2013). Critical service logic: Making sense of value creation and co-creation. Journal of the Academy of Marketing Science, 41(2), 133-150. 
Gummesson, E., \& Mele, C. (2010). Marketing as value co-creation through network interaction and resource integration. Journal of Business Market Management, 4(4), 181198.

Hair, J. F., Black, W. C., Babin, B. J., \& Anderson, R. E. (2010). Multivariate data analysis: A global perspective (7th ed.). Upper Saddle River, NJ: Pearson.

Heinonen, K., \& Strandvik, T. (2015). Customer-dominant logic: Foundations and implications. Journal of Services Marketing, 29(6/7), 472-484.

Hobfoll, S. E., Halbesleben, J., Neveu, J.-P., \& Westman, M. (2018). Conservation of resources in the organizational context: The reality of resources and their consequences. Annual Review of Organizational Psychology and Organizational Behavior, 5, 103-128.

McColl-Kennedy, J. R., Snyder, H., Elg, M. H., Witell, L., Helkkula, A., Hogan, S., \& Anderson, L. (2017). The changing role of the health care customer: Review, synthesis and research agenda. Journal of Service Management, 28(1), 2-33.

Nguyen, H. L. (2018). The role of customer operant resources in health care value creation. Service Business, 13(5), 1-22.

Nguyen, H. L., Pham, A. N. T., \& Pham, T. N. (2016). The effects of interaction behaviors of service frontliners on customer participation in the value co-creation: A study of health care service. Service Business, 1-25.

Ostrom, A. L., Bitner, M. J., Brown, S. W., Burkhard, K. A., Goul, M., Smith-Daniels, V. L., ...Rabinovich, E. (2010). Moving forward and making a difference: Research priorities for the science of service. Journal of Service Research, 13(1), 4-36.

Sharma, N., \& Patterson, P. G. (1999). The impact of communication effectiveness and service quality on relationship commitment in consumer, professional services. Journal of Services Marketing, 13(2), 151-170.

Vargo, S. L., \& Lusch, R. F. (2004). Evolving to a new dominant logic for marketing. Journal of Marketing, 68(1), 1-17.

Vargo, S. L., \& Lusch, R. F. (2008). Service-dominant logic: Continuing the evolution. Journal of the Academy of Marketing Science, 36(1), 1-10.

Vargo, S. L., \& Lusch, R. F. (2017). Service-dominant logic 2025. International Journal of Research in Marketing, 34(1), 46-67.

Yi, Y., \& Gong, T. (2013). Customer value co-creation behavior: Scale development and validation. Journal of Business Research, 66(9), 1279-1284. 